\title{
Ultrasonic detection of spall damage nucleation under low-velocity repeated impact
}

\author{
N. Nishimura ${ }^{1}$, K. Murase ${ }^{1}$, T. Ito ${ }^{2}$, and T. Watanabe ${ }^{3}$ \\ ${ }^{1}$ Meijo University, Department of Vehicle and Mechanical Engineering, 1-501 Shiogamaguchi, Tempaku-ku, \\ Nagoya 468-8502, Japan \\ 2 Nagoya Institute of Technology, Department of Mechanical Engineering, Gokisocho, Showa-ku, \\ Nagoya 466-8555, Japan \\ ${ }^{3}$ Graduate Student, Meijo University, Department of Transportation Engineering, 1-501 Shiogamaguchi, \\ Tempaku-ku, Nagoya 468-8502, Japan
}

\begin{abstract}
Repeated plate impact testing with impact stress well below the threshold spall-stress $(2.6 \mathrm{GPa})$ on medium carbon steel was carried out to the identical target plate by impacting the flyer plate. Occurrence of spall damage under low-velocity repeated impact was evaluated nondestructively with a low frequency scanning acoustic microscope. We observed the spall damage distribution by the B- and C-scan images. In order to initiate the spall damage (voids in a ductile material or cracks in a brittle one) the particular value of threshold spall-stress should be exceeded what already belongs to a commonly accepted knowledge. Generally, the spall damage development is dependent on the amplitude and the duration of the stress pulse. If the stress is high and duration is long enough to create tensile failure of material, the voids or cracks nucleate along the spall plane, and consequently, they form macrocracks. Therefore, the spall damage does not create when the first impact stress is less than the threshold spall-stress. However, after the fifth low-velocity repeated impact test, the generation of the spall damage was detected, even if the impact stress (1.1-1.7 GPa) was lower than the threshold spall-stress (2.6 GPa).
\end{abstract}

\section{Introduction}

Spall damages, microvoids or microcracks, are nucleated by an intensive tensile stress pulse within a solid that has been impacted at high velocity. Spall damage is invisible, because it appears within a solid. Traditionally the extent of spall damage has been examined by destructive means, namely sectioning and polishing the sample and observation with an optical or scanning electron microscope [1-3]. However these destructive methods are applied to neither actual structural parts nor investigation of the spall damage growth under repeated impacts on the identical sample.

For the nondestructive evaluation of the spall damage, we should know the size and the quantity of the minute damage as well as the location. Among many nondestructive methods, an ultrasonic method is versatile and suitable for quantitative evaluation. The present authors already demonstrated effectiveness of the non-destructive ultrasonic method for the evaluation of the spall damage and recovery in aluminum and medium carbon steel plates under repeated impacts [4]. The evolution/recovery of spall damage was examined by low frequency scanning microscopy and ultrasonic measurement.

Generally, in order to initiate the spall damage the first impact stress should be exceeded the particular value of threshold spall-stress [1,2]. However, it is necessary to examine that the spall damage is generated by impacting repeatedly with the lower impact stress than the threshold, such as the fatigue phenomenon. The aim of the present paper is to reveal with ultrasonic technique the nucleation of the spall damage under low-velocity repeated impacts on the identical target plate of medium carbon steel.

\section{Spallation under plate impact experiment}

Spall damage, i.e., microvoids or microcracks, is nucleated within a target plate by triaxial tensile stress pulse, which is generated by the plate impact experiment. When a target plate is impacted by a flyer plate at high velocity, the compressive stress waves generated in both plates travel toward the free surfaces. The waves are reflected at the free surfaces with the reversed phase, namely tensile nature, then the reflected waves travel back toward the impacted surface with canceling the compressive wave. Thus it is called as rarefaction wave. When rarefaction waves within target plate propagated from impacted and free surfaces superpose, a tensile stress pulse is generated. Generally, the spall damage development is dependent on the amplitude and the duration of the tensile stress pulse [1,2]. If the impact stress is higher than the threshold spall stress and duration is long enough to create tensile failure of material, the spall damage is nucleated in the narrow area called the spall plane, and consequently, they form macrocracks.

\section{Experimental methods}

\subsection{Plate impact experiments}

Target plate made of medium carbon steel ( $0.45 \%$ carbon) was impacted repeatedly by flyer plate of the same material (the detailed characteristics are given in table 1). The detailed listing of the conditions of the experiments carried out on different specimens is provided in table 2. The dimensions of the used materials and schematic of the experimental setup is presented in figure 1. The parallel 
Table 1. Material parameters of the investigated materials.

\begin{tabular}{|l|l|}
\hline Material & Carbon steel \\
\hline Density $\rho\left[\mathrm{kg} / \mathrm{m}^{3}\right]$ & 7790 \\
\hline Poisson's ratio $v$ & 0.29 \\
\hline Young's modulas $E[\mathrm{GPa}]$ & 209 \\
\hline Bulk modulus $K[\mathrm{GPa}]$ & 164 \\
\hline Shear modulus $G[\mathrm{GPa}]$ & 81 \\
\hline Bulk wave velocity $C[\mathrm{~m} / \mathrm{s}]$ & 5920 \\
\hline
\end{tabular}

Table 2. The detailed listing of the test conditions carried out on medium carbon steel specimens and the occurrence of spall damage after the each impact detected by SLFAM-technique.

\begin{tabular}{|c|c|c|c|c|c|l|}
\hline \multicolumn{2}{|c|}{ Specimen No. } & \multicolumn{5}{|c|}{ Number of Impact } \\
\cline { 3 - 7 } \multicolumn{2}{|c|}{ R2 } & 1 st & 2nd & 3 rd & 4 th & 5 th \\
\hline \multirow{3}{*}{ Impact velocity $V[\mathrm{~m} / \mathrm{s}]$} & 58 & 73 & 66 & 56 & 55 \\
\cline { 2 - 7 } & Impact stress $\sigma[\mathrm{GPa}]$ & 1.3 & 1.7 & 1.5 & 1.3 & 1.3 \\
\cline { 2 - 7 } & Spall Damage & $\times$ & $\times$ & $\times$ & $\bigcirc$ & $\bigcirc$ \\
\hline \multirow{4}{*}{ R6 } & Impact velocity $V[\mathrm{~m} / \mathrm{s}]$ & 52 & 60 & 47 & 65 & 64 \\
\hline \multirow{4}{*}{ R7 } & $\begin{array}{c}\text { Impact stress } \\
\sigma[\mathrm{GPa}]\end{array}$ & 1.2 & 1.4 & 1.1 & 1.5 & 1.5 \\
\cline { 2 - 7 } & Spall Damage & $\times$ & $\times$ & $\times$ & $\times$ & $\bigcirc$ \\
\cline { 2 - 7 } & $\begin{array}{c}\text { Impact velocity } \\
V[\mathrm{~m} / \mathrm{s}]\end{array}$ & 56 & 51 & 52 & 64 & 75 \\
\cline { 2 - 7 } & Impact stress $\sigma[\mathrm{GPa}]$ & 1.3 & 1.2 & 1.2 & 1.5 & 1.7 \\
\hline & Spall Damage & $\times$ & $\times$ & $\times$ & $\times$ & $\bigcirc$ \\
\hline
\end{tabular}

The symbols $\bigcirc$ and $\times$ stand for distinguished and non- identified spall damage, respectively.

orientation of the target and flyer plates was exactly satisfied in our experiments (the deviation should not exceed $1 \mathrm{mrad}$ as stated in ref. [5]).

The flyer plate bonded to the plastic sabot was accelerated by a single stage gas gun. The target plate was also bonded to the plastic stage fastened to the target holder and velocity measurement unit (details in figure 1). The velocity of the flyer was measured by the optical fiber switches located 20 and $30 \mathrm{~mm}$ away from the target plate. The special recovery chamber has been constructed to recover the target plate with the smallest possible external deformation [4]. The front and rear surfaces of the target plate were polished with a grinder or the \#1500 emery paper after each impact test. Repeated impacts were given to the identical target plate with different low impact velocities (see table 2).

When the target and flyer are same material, the compressive stress $\sigma$ at the impacted surface induced just after the collision can be obtained from the following relationship $[4,6,7]$ :

$$
\sigma=\frac{1}{2} \rho C V, \quad C=\sqrt{\frac{1}{\rho}\left(K+\frac{4}{3} G\right)},
$$

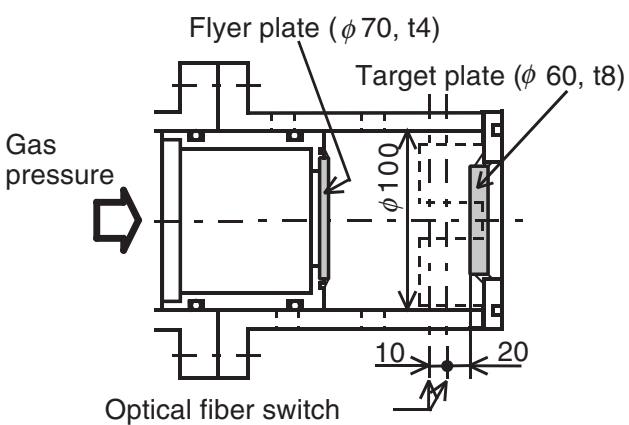

Fig. 1. Location of the target, flyer and the 'velocity measurement unit' denoted in the schematic of the expermiental setup. The exact dimensions used in our experiments are indicated in the figure.

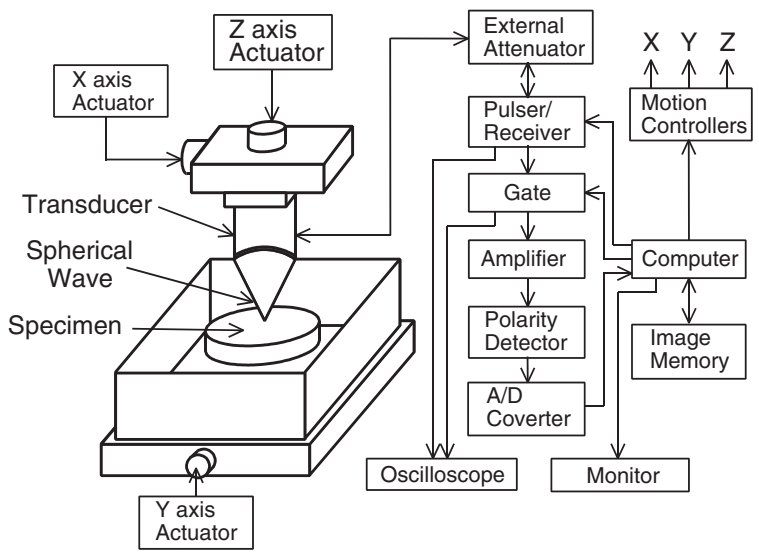

Fig. 2. The clarification of our acoustic microscopy provided in a graphical form.

where $\rho, C, V, K$ and $G$ are the density, the bulk (elastic) wave velocity, the velocity of the flyer, the bulk elastic modulus and the shear elastic modulus of an investigated material.

\subsection{C- and B-scan Imaging}

In order to detect the spall damage occurrence, we used the scanning low frequency acoustic microscopy (SLFAM, Olympus UH Pulse-100 - the schematic given in figure 2, see also refs. [9-11]). The scanning acoustic images were obtained by detection of the amplitude of the longitudinal wave reflected from the elemental area of the specific defects such as inclusions, voids or cracks. The signal intensity registered at each point was mapped as a set of spots of variable brightness (e.g. figure 4 in the Results chapter).

The spall damage was examined by ultrasonic $\mathrm{C}$ - or B-scan images that enabled us to visualize void/crack distributions when viewed parallel and perpendicular to an impact plane. In a greater detail, the pertinent experimental parameters [12] such as the central frequency of $30 \mathrm{MHz}$, beam diameter at focus equal $0.28 \mathrm{~mm}$ and focal length (in water) of $25.4 \mathrm{~mm}$ were achieved with the PolyVinylidene DiFluoride (PVDF) point-focus transducer. It provides clear images of voids or cracks at arbitrary depth by setting the gate width and the gate position from the received wave 


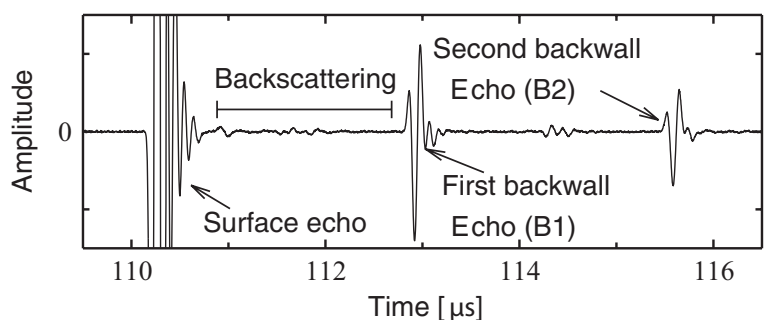

Fig. 3. The typical waveform recorded using the ultrasonic measurement.

reflected at impact surface [4]. This advantage of PVDF transducer makes him very convenient for examining thick specimens with the internal damage.

To assure the absence of pre-existing voids or cracks, all specimens were inspected by SLFAM before the initial impact test. C-scan images were acquired at the 2.0, 2.5 and 3.0 to $4.0 \mathrm{~mm}$ depth in the $0.1 \mathrm{~mm}$ interval from the impact surface and B-scan images were taken at the central cross section of the target plate with rigorously kept conditions (the constant amplitude gain, brightness, contrast etc.). The gate width for detecting the signal intensity was fixed at $30 \mathrm{~ns}$ (about $90 \mu \mathrm{m}$ thickness in the medium carbon steel).

\subsection{Quantitative evaluation of spall damage}

In order to quantitatively evaluate the spall damage evolution we determined experimentally a set of pertinent parameters that includes: time-of-flight (longitudinal wave velocity), backwall echo height of target plates (for explanation please find the B1 and B2 parameters in figure 3) and the wave intensity backscattered at spall damage. The employed digital ultrasonic measurement system [13] is composed of ultrasonic pulser/receiver, A/D converter board and personal computer. The longitudinal wave transducer with the $10 \mathrm{MHz}$ frequency and $6.4 \mathrm{~mm}$ diameter made it possible to record the wave from the back wall and backscattering echoes at three different locations (in the center as well as $10 \mathrm{~mm}$ away), all of these before and right after the impact test.

The careful analysis [details in ref. [4]] of the registered waveforms (depicted in figure 3) enabled us to conclude on the ultrasonic velocity and attenuation (amplitude ratio of B2 to B1 echo). The frequency-dependent backscattering intensity, characterized by the nondimensional value $N$ :

$$
N=\int_{f_{1}}^{f_{2}} B(f) d f \mid \int_{f_{1}}^{f_{2}} U(f) d f
$$

where $B(f)$ defines the amplitude spectrum of the backscattering wave, $U(f)$ stands for the surface echo, while $f_{1}$ and $f_{2}$ denote the lower and upper limit of the range of frequency $f$, namely 3 and $14 \mathrm{MHz}$ [4]. The target plates were subjected to detailed examination (C- and B-scan imaging, the measurements of ultrasonic velocity, attenuation and backscattering intensity) after the consecutive impact tests.

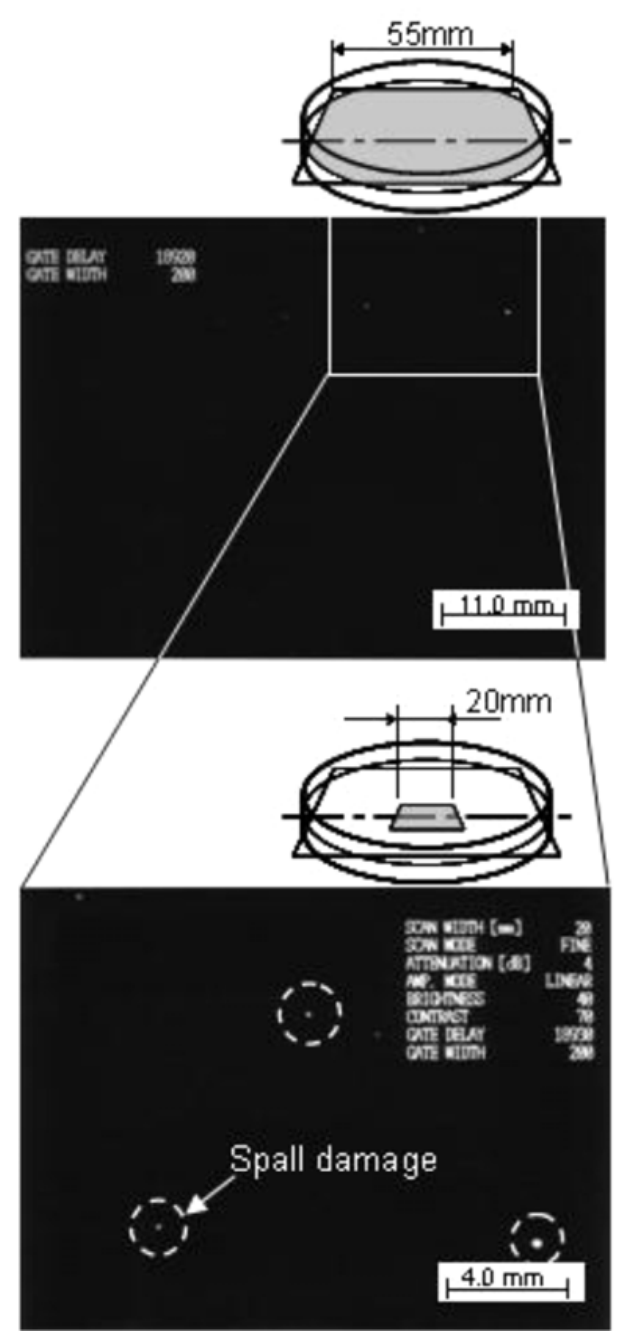

Fig. 4. The spall damage in medium carbon steel generated after the fifth impact during low-velocity repeated impact tests. History of impact stress are 1.2 GPa (first), 1.4 GPa (second), 1.2 GPa (third), 1.5 GPa (fourth) and 1.5 GPa (fifth). The exposed particular data (C-scan image at the $3.3 \mathrm{~mm}$ depth) were registered for the sample No.R6.

\section{Results and discussion}

\subsection{Detection of spall damage under low-velocity repeated impacts}

The present authors made effort to evaluate the threshold stress that results in generating of spall damage in the investigated metals [4]. It was found that the medium carbon steel requires $2.6 \mathrm{GPa}$ to initiate the spall damage. The detailed account for the impact test conditions and the stage when flaws were detected is given in table 2 . The evaluation of the impact stress pertinent to our study was accomplished using the general formula (Eq. (1)) with the proper materials' parameters (table 1) and flyer's velocities (tables 2). In the every impact tests, impact stress was given to target plate less than the threshold spall-stress.

As a result of the careful observation by the acoustic microscope, the spall damage was detected after the fifth impact [figure 4 (spec. No. R6), White part in the image 
(a)
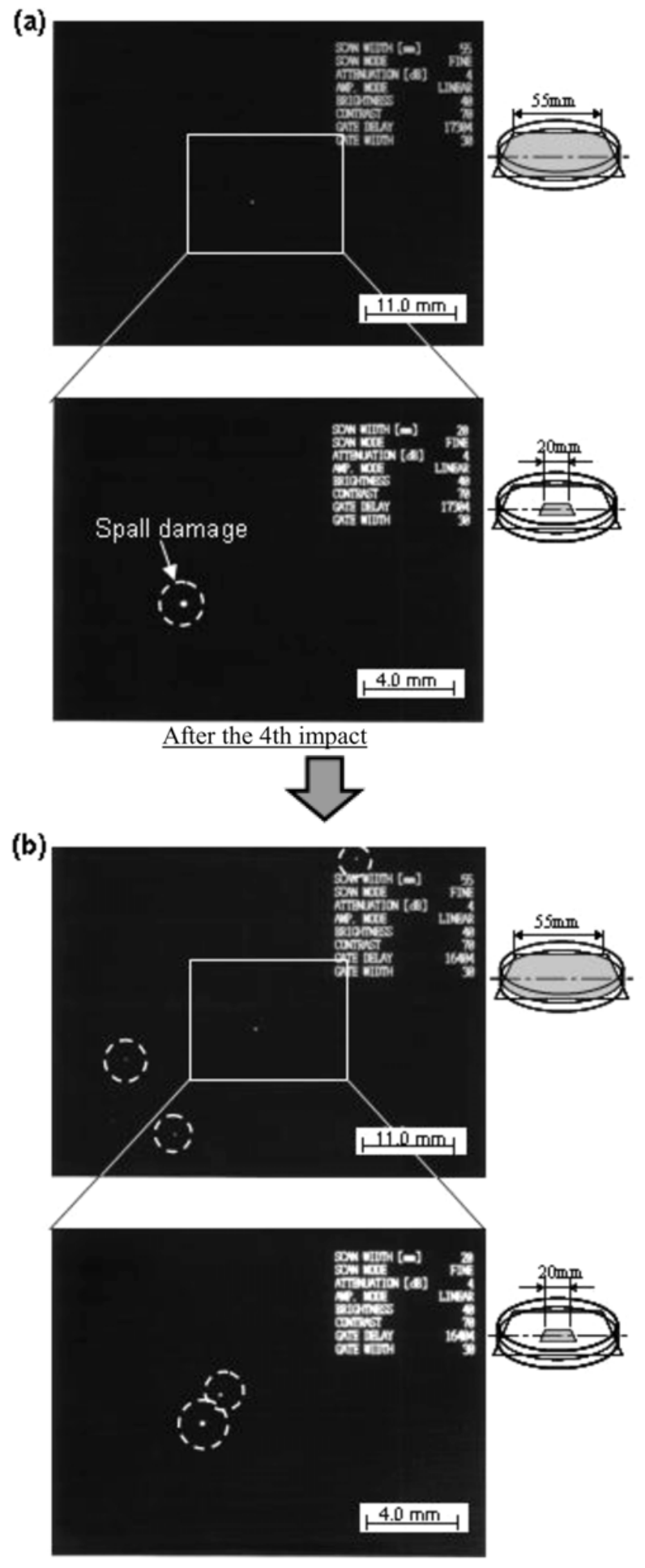

After the 5th impact

Fig. 5. The spall damage in medium carbon steel generated after the fourth (a) and fifth (b) impact during low-velocity repeated impact tests. History of impact stress are $1.3 \mathrm{GPa}$ (first), 1.7 GPa (second), 1.5 GPa (third), 1.3 GPa (fourth) and 1.3 GPa (fifth). The exposed particular data (C-scan image at the $3.5 \mathrm{~mm}$ depth) were registered for the sample No.R2. The spall damage nucleated by the fourth impact was increased by the fifth impact.

indicates the defect by reflected signal]. And, in the case of another specimen, the spall damage detected after the fourth impact was increased by the fifth impact [figure 5

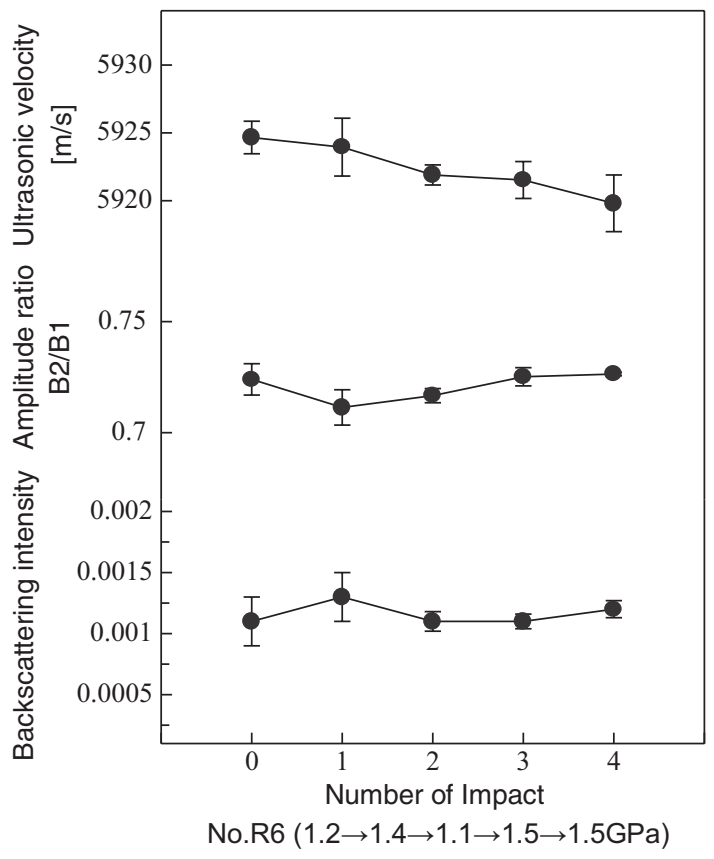

Fig. 6. Changes of ultrasonic velocity, amplitude ratio and backscattering intensity during the low-velocity repeated impact test. The data correspond to $\mathrm{C}$-scan images displayed in the figure 4 .

(spec. No. R2), It is worth emphasizing that the images refer to the exactly same region of target plate]. Namely, the spall damage is generated by the repeated impact, even if the impact stress was lower than the threshold spallstress. Nucleation of the spall damage will concerns the history of impact testing and its influence on mechanical behavior.

\subsection{Change in ultrasonic velocities, attenyation and backscattering intensity}

The wave velocity in solids containing a large number of voids or cracks is generally lower than this measured for defect-free materials. The difference stems from the ultrasonic wave scattering at voids or cracks. Moreover, the presence of indicated defects enhances attenuation and the backscattering intensity. Consequently, the ultrasonic wave velocity, attenuation (amplitude ratio, B2/B1) and backscattering intensity is related to spall damage $[4,8]$ and therefore we use it to estimate this very phenomenon.

The measurement of the pertinent ultrasonic characteristics was carried out on samples that were exposed to lowvelocity repeated impacts (e.g., for No. R6). Especially in the ultrasonic characteristics, the ultrasonic velocity slightly decreases with the increase in the impact number, as shown in figure 6. However, it was not possible to obtain the clear change of each ultrasonic variables of the every impact test for the quantitative evaluation of the damage, owing to the extremely minute and small amount of the spall damage nucleated in the specimen. These authors will make an attempt other ultrasonic methods for quantitative evaluation of generation of smaller, microscopic defects, 
for instance, usage of the nonlinear ultrasonic method [14] would be a better choice.

\section{Conclusions}

The nucleation of the spall damage generated in medium carbon steel $(0.45 \% \mathrm{C})$ by low-velocity repeated impact testing was evaluated by means of the nondestructive, ultrasonic method (the ultrasonic C-scan images as well as the ultrasonic velocity, the attenuation and the backscattering intensity). After the fifth low-velocity repeated impact test, the generation of the spall damage was detected by $\mathrm{C}$-scan image observation, even if the impact stress (1.1-1.7 GPa) was lower than the threshold spall-stress. Nucleation and increase of spall damage are dependent on the history of the impact stress given to the specimen.

\section{References}

1. L. Davison, R. A. Graham, Phys. Rep. 55, 4 (1979)

2. M. A. Meyers, C. T. Aimone, Progr. Mater. Sci. 28 (1983)
3. D. R. Curran, L. Seaman, D. A. Shockey, Phys. Rep. 147, 5-6 (1987)

4. N. Nishimura, K. Murase, T. Ito, R. Nowak, Int. J. Impact Eng. 38, 4 (2011)

5. Y. Oved, G. E. Luttwak, J. Comp. Mat. 12 (1978)

6. T. Hayashi, Y. Tanaka, Impact Engineering, (Nikkan Kogyo Shimbun Ltd. 1988)

7. J. A. Zukas, High Velocity Impact Dynamics, (A Wiley-Interscience Publication: John Wiley \& Sons, INC. 1990)

8. K. Kawashima, Trans. of JSME-A , 67, 655 (2001)

9. R. S. Gilmore, K. C. Tam, J. D. Young, D. R. Howard, Phil. Trans. R Soc. London. A320 (1986)

10. B. Tittmann, C. Miyasaka, H. Kasano, in Proceedings of ECNDT 2006, Th.4.7.4.

11. M. Takada, M. Haccho, M. Hayashi, F. Uchino, Optical and Electro-Optical Engineering Contact. 27, 4 (1989)

12. G. S. Kino, Acoustic Waves: Devices, Imaging, and Analog Signal Processing, (Prentice-Hall. 1987)

13. K. Kawashima, I. Fujii, Review of Progress in QNDE. 14 (1995)

14. K. Kawashima, J. Okada, K. Nawa, N. Nishimura, Review of progress in QNDE. 20 (2001) 